HNO 2011 · 59:857-858

DOI 10.1007/s00106-011-2360-6

Online publiziert: 22. Juli 2011

(c) Springer-Verlag 2011

\section{A. Dietz}

Klinik und Poliklinik für Hals-, Nasen-, Ohrenheilkunde, Universitätsklinikum Leipzig

\title{
Aussichtsreiche Ansätze translationaler Forschung in der Kopf-Hals-Onkologie
}

Betrachtet man die Felder unseres Faches, in denen sich Forschung nicht nur lohnt, sondern zu unseren dringendsten Entwicklungsaufgaben gehört, so gehören unstrittig die Plattenepithelkarzinome des Kopf-Hals-Bereichs („head and neck squamous cell carcinoma“, HNSCC) auf einen der vordersten Ränge. Mehr als die Hälfte unserer Patienten mit HNSCC stirbt an den Folgen ihrer Erkrankung. Die aktuellen Daten der EUROCARE4-Analysen dokumentieren in ernüchternder Weise die 5-Jahres-Überlebensraten von HNSCC-Patienten in Europa mit Erstdiagnose in den Jahren 1995-1999 [1]. Hier ergibt sich für alle berücksichtigten Kopf-Hals-Krebs-Patienten (49.569 Fälle) ein relatives Überleben von $42 \%$ und dies nahezu unverändert in den letzten 30 Jahren.

Parallel hat sich die klinische Onkologie im Generellen auf vielen Ebenen weiterentwickelt. Für unser Fach bedeutsam sind neben den hoch differenzierten operativ-rekonstruktiven Verfahren die Weiterentwicklungen der Strahlentherapie (intensitätsmodulierte Radiotherapie, IMRT; Schwerionenbestrahlung) und die Chemotherapie/"Targeted-Therapie" (ESMO-Guidelines 2009). Angeregt durch die zunehmende Kompetenzzentrumsbildung nach den Vorgaben der Deutschen Krebsgesellschaft (zertifiziertes Kopf-Hals-Modul) bemühen wir uns um interdisziplinäre Therapieplanung in Tumorboards und verständigen uns überwiegend auf Basis weniger kontrollierter Studien und einer umfangreichen empirischen Tradition über das jeweils sinnvolls- te Behandlungskonzept. Hierbei wird immer wieder deutlich, dass wir nur spärlich verifizierte Konzepte für den einzelnen Tumorpatienten bereithalten, da der individuellen, hoch heterogenen Tumorbiologie, der im Einzelfall möglichen guten Response gegenüber multimodalen Therapieoptionen und der interindividuellen drastischen Unterschiede im Ergebnis bislang im Vorfeld keine Rechnung getragen werden kann. Letztlich sind daher primär chirurgische Konzepte, soweit eine komplette Resektion (Ro) möglich ist, die aufgrund der wenig kalkulierbaren Dynamik eines HNSCC sichersten Verfahren mit dem größten Vertrauensvorschuss.

\section{> Tumorbiologische Faktoren spielen faktisch keine Rolle für die Therapieentscheidung}

Für unsere Therapieentscheidung ziehen wir derzeit lediglich das TNM-Stadium, den Resektionsgrad und die Tumorlokalisation in Betracht. Tumorbiologische Faktoren spielen faktisch keine Rolle. Das vorliegende Themenheft versucht anhand einiger ausgewählter Topics einerseits Licht in die aktuellen Möglichkeiten und Ergebnisse molekularbiologischer Forschung zu bringen und andererseits einige wenige Aspekte aufzuzeigen, die unsere translationale klinische Forschung in Zukunft weiter bringen sollen.

Beispielhaft für einen mittlerweile kurz vor der klinischen Routinebetrachtung stehenden molekularen Marker, der sich durch translationale Forschung herauskristallisierte, hat Klussmann den Surro- gatmarker p16 auf dem Boden des HPV16induzierten HNSCC näher unter die Lupe genommen. Da p16 der aktuell größte Hoffnungsträger für eine individualisierte Therapie bei den Oropharynxkarzinomen darstellt, lohnt es sich, die Thematik etwas näher zu betrachten. Außerdem ist die Diskussion über die Tumorstammzellen anzusprechen, die komplett neue Ansätze der Tumorentstehung, Therapieresistenz und Progression eines HNSCC hervorgebracht hat.

Mozet hat sich in seinem Beitrag dieser Frage gewidmet und die aktuelle Daten- und Hypothesenlage des Tumorstammzellkonzepts bei HNSCC zusammengestellt. Natürlich wirft dieses Thema eine Vielzahl von Fragen auf, die mit jeder vermeintlichen Eingrenzung durch neue Identifikatoren von Tumorstammzellen eher zunehmen. In besonderer Weise sieht sich die aktuelle translationale Forschung in diesem Kontext einer fast täglich wachsenden Zahl von Regulatoren, Pathways, Pathway-Kommunikatoren konfrontiert, die durch die neuen Omics- bzw. systembiologischen Techniken generiert werden. Diese Datenmengen aus Gen-Profiling-Studien berühren die neue Thematik der korrelierten Gensets. Hierunter versteht man Korrelationsanalysen von multiplen Daten aus Arrays bzw. diverse Chiptechniken (Omics) mit klinischen Verlaufsparametern. Die hieraus resultierenden prognostischen Genlisten könnten neue prognostische Markergenerationen (genetisches Subtyping) beschreiben, die uns eine detailliertere Betrach- 
tung der individuellen Tumorbiologie ermöglichen könnten.

Aktuell kämpfen aber zahlreiche Biometriker an der sinnvollen Kalkulation solcher Datensätze und scheitern an den kleinen Fallzahlen, der mangelnden Reproduzierbarkeit innerhalb gleicher Kohorten und den immensen Kosten. Der Autor hat sich mit dieser Thematik des Einsatzes korrelierter Gensets in der translationalen Forschung des HNSCC in seinem Beitrag befasst. Darüber hinaus werden die neuen Notwendigkeiten eines standardisierten Biobankings und Grenzbereiche sinnvoller translationaler Forschung in der Onkologie unseres Fachgebiets beleuchtet.

\section{(7) Der Reiz der Ex-vivo-Testung eines Tumors liegt in der unmittelbar messbaren Reaktion}

Schließlich führen die Schwierigkeiten bei den Gensets und Markern zu Ansätzen der Gesamtbetrachtung des zellulären Tumors. Nicht die genetischen Variablen, sondern das simulierte biologische Verhalten der Tumorzellen (Tumorzellverband) könnte im translationalen Kontext mehr Aufmerksamkeit gewinnen. Hierzu gibt es eine Reihe neuerer Testsysteme, die von Wichmann in seinem Beitrag beschrieben werden. Der Reiz einer solchen Ex-vivo-Testung des individuellen Tumors im Rahmen einer Kurzzeit-Primärkultur aus einer Biopsie liegt in der unmittelbar messbaren Reaktion des Tumors, beispielsweise auf eine Chemotherapie. Verschiedene Verfahren sind mittlerweile sehr ausgereift und bieten insbesondere für das HNSCC aufgrund der enormen Heterogenität bezüglich des Therapieansprechens sinnvolle Ansätze für die translationale Einbettung der Responseevaluation innerhalb klinischer Studien.
Die in diesem Themenheft ausgewählten Beiträge richten sich aus Sicht der Autoren schlaglichtartig auf einige relevante Themen für die zukünftige translationale Forschung in der Kopf-Hals-Onkologie - ohne Anspruch auf Vollständigkeit. Die Abhandlungen sollen deutlich machen, dass wir HNO-Ärzte die Herausforderungen, die sich durch die neuen technischen Möglichkeiten ergeben, kompetent und interdisziplinär vernetzt annehmen müssen, um die Onkologie unseres Fachs nachhaltig weiterzuentwickeln. Ich hoffe, dass die Auswahl der Beiträge Ihr Interesse findet.

\section{Mit kollegialen Grüßen}

Ihr

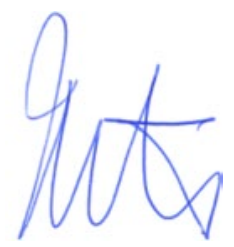

Prof. Dr. Andreas Dietz

\section{Korrespondenzadresse Prof. Dr. A. Dietz}

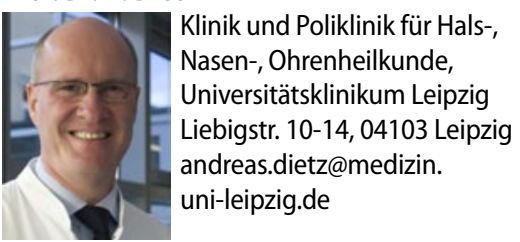

\section{Literatur}

1. Sant M, Allemani C, Santaquilani M, Knijn A, Marchesi F, Capocaccia R; EUROCARE Working Group (2009) EUROCARE-4. Survival of cancer patients diagnosed in 1995-1999. Results and commentary. Eur J Cancer 45(6):931-991

\section{Orbitatumore und -rekonstruktion}

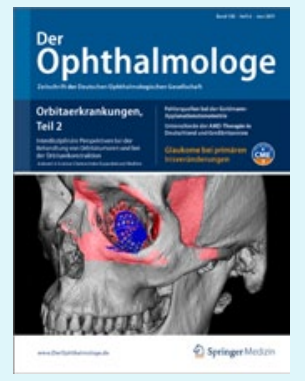

Orbitatumore

sind Tumore der Augenhöhle. Sie verursachen Doppelbilder, Druckgefühl, das Hervortreten des Auges und beeinträchtigen unter Umständen den

Sehnerv. Die Fortsetzung des Leitthemas "Orbitaerkrankungen" widmet sich der Behandlung von Orbitatumoren und der Rekonstruktion der Orbita nach funktionsund formverändernden Vorerkrankungen. Dabei wird auf klinische Diagnostik und radiologische Abklärung, die Therapie von Orbitatumoren und auch speziellen Aspekte von Orbitatumoren im Kindesalter eingegangen. Die Ausgabe 6/2011 von Der Ophthalmologe legt hier folgende Schwerpunkte:

- Klinische und neuroradiologische Diagnostik bei Orbitatumoren

- Therapie anterior gelegener Orbitatumoren

- Therapie posterior gelegener Orbitatumoren

- Funktionserhaltende Rekonstruktion nach Orbitatrauma

Bestellen Sie diese Ausgabe zum Preis von $34,-€$ bei Springer Customer Service Center Kundenservice Zeitschriften

Haberstr. 7

69126 Heidelberg

Tel.: +49 6221-345-4303

Fax: +49 6221-345-4229

E-Mail: leserservice@springer.com

P.S. Vieles mehr rund um Ihr Fachgebiet finden Sie auf www.springermedizin.de 